\title{
Lacunary Statistical Convergence in Fuzzy Normed Linear Spaces
}

\author{
Muhammed Recai Turkmen", , Muhammed Cinar ${ }^{2}$ \\ ${ }^{1}$ Department of Mathematics, Afyon Kocatepe University, Afyon, Turkey \\ ${ }^{2}$ Department of Mathematics, Mus Alparslan University, Mus, Turkey
}

Email address:

mrtmath@gmail.com (M. R. Turkmen), muhammedcinar23@hotmail.com (M. Cinar)

${ }^{*}$ Corresponding author

\section{To cite this article:}

Muhammed Recai Turkmen, Muhammed Cinar. Lacunary Statistical Convergence in Fuzzy Normed Linear Spaces. Applied and Computational Mathematics. Vol. 6, No. 5, 2017, pp. 233-237. doi: 10.11648/j.acm.20170605.13

Received: July 19, 2017; Accepted: August 17, 2017; Published: October 23, 2017

\begin{abstract}
In this paper, it is introduced the concept of lacunary statistical convergence with respect to a fuzzy norm by using lacunary statistical convergence of a sequence and statistical convergent of a sequence with respect to fuzzy norm. It also has studied the relation between these concepts.
\end{abstract}

Keywords: Lacunary Statistical Convergence, Fuzzy Normed Linear Space, Sequences Space

\section{Introduction}

The concept of statistical convergence of real number sequences was introduced Fast [7] and Steinhaus [18] and later reintroduced by Schoenberg [15] independently. Many mathematicians such as Connor [5], Fridy [9], Colak [4], Et and Cinar [3], T. Šalát [19] etc studied the concept of statistical convergence in summability theory.

A complex number sequence $x=\left(x_{k}\right)$ is said to be statistically convergent to number $L$ if for every $\varepsilon>0$,

$$
\lim _{n} \frac{1}{n}\left|\left\{k \leq n:\left|x_{k}-L\right| \geq \varepsilon\right\}\right|=0, \text { [9] }
$$

where the vertical bars indicate the number of elements in the enclosed set. In this case, it is written as $S-\lim x=L$ or $x_{k} \rightarrow L(S)$. It will also used $S$ to denote the set of all statistically convergent sequences. Cesaro summability, which is a natural relationship with statistical convergence, is defined by

$$
[C, 1]=\left\{x=\left(x_{k}\right): \lim _{n \rightarrow \infty} \frac{1}{n} \sum_{k=1}^{n}\left|x_{k}-L\right|=0, \text { for some } L\right\}
$$

Let $(X,\|\|$.$) be an FNS. A sequence \left(x_{k}\right)$ in $X$ is statistically convergent to $L \in X$ with respect to the fuzzy norm on $X$ provided that for each $\varepsilon>0$, we have

$$
\delta\left(\left\{k \in \mathbb{N}: D\left(\left\|x_{k}-L\right\|, \tilde{0}\right) \geq \varepsilon\right\}\right)=0
$$

This implies that for each $\varepsilon>0$, the set $K(\varepsilon)=\left\{k \in \mathbb{N}:\left\|x_{k}-L\right\|_{0}^{+} \geq \varepsilon\right\}$ has natural density zero; namely, for each $\varepsilon>0,\left\|x_{k}-L\right\|_{0}^{+}<\varepsilon$ for almost all $\mathrm{k}$ [17].

If $\theta=\left\{k_{r}\right\}$ is a lacunary sequence then we will take an increasing integer sequence such that $k_{0}=0$ and $h_{r}=k_{r}-k_{r-1} \rightarrow \infty$ as $r \rightarrow \infty$. Throughout this paper, the intervals determined by $\theta$ will be shown as $I_{r}=\left(k_{r-1}, k_{r}\right]$ and the ratio $\frac{k_{r}}{k_{r-1}}$ will be abbreviated by $q_{r}$.

The set of all Lacunary summable sequence is shown by $N_{\theta}$, which is defined by

$$
N_{\theta}=\left\{x: \lim _{r \rightarrow \infty}\left(\frac{1}{h_{r}} \sum_{k \in I_{r}}\left|x_{k}-L\right|\right)=0, \text { for some } L\right\} \quad[16]
$$

Fuzzy sets are considered with respect to a nonempty base set $X$ of elements of interest. The essential idea is that each 
element $x \in X$ is assigned a membership grade $u(x)$ taking values in $[0,1]$, with $u(x)=0$ corresponding to nonmembership, $0<u(x)<1$ to partial membership, and $u(x)=1$ to full membership. According to Zadeh [21] a fuzzy subset of $X$ is a nonempty subset $\{(x, u(x)): x \in X\}$ of $X \times[0,1]$ for some function $u: X \rightarrow[0,1]$. The function $u$ itself is often used for the fuzzy set.

A fuzzy set $u$ on $\mathbb{R}$ is called a fuzzy number if it has the following properties:

1. $u$ is normal, that is, there exists an $x_{0} \in \mathbb{R}$ such that $u\left(x_{0}\right)=1$;

2. $u$ is fuzzy convex, that is, for $x, y \in \mathbb{R}$ and $0 \leq \lambda \leq 1$, $u(\lambda x+(1-\lambda) y) \geq \min [u(x), u(y)]$

3. $u$ is upper semicontinuous;

4. suppu $=\operatorname{cl}\{x \in \mathbb{R}: u(x)>0\}$, or denoted by $[u]_{0}$, is compact.

Let $L(\mathbb{R})$ be set of all fuzzy numbers. If $u \in L(\mathbb{R})$ and $u(t)=0$ for $t<0$, then $u$ is called a non-negative fuzzy number. We write $L^{*}(\mathbb{R})$ by the set of all non-negative fuzzy numbers. We can say that $u \in L^{*}(\mathbb{R})$ iff $u_{\alpha}^{-} \geq 0$ for each $\alpha \in[0,1]$. Clearly we have $\tilde{0} \in L(\mathbb{R})$. For $u \in L(\mathbb{R})$, the $\alpha$ level set of $u$ is defined by

$$
[u]_{\alpha}=\left\{\begin{array}{cc}
\{x \in \mathbb{R}: u(x) \geq \alpha\}, & \text { if } \alpha \in(0,1] \\
\text { suppu, } & \text { if } \alpha=0 .
\end{array}\right.
$$

A partial order $\preceq$ on $L(\mathbb{R})$ is defined by

$u \preceq v$ if $u_{\alpha}^{-} \leq v_{\alpha}^{-}$and $u_{\alpha}^{+} \leq v_{\alpha}^{+}$for all $\alpha \in[0,1]$

Arithmetic operation $\oplus, \ominus, \odot$ and $\oslash$ on $L(\mathbb{R}) \times L(\mathbb{R})$ are defined by

$$
\begin{aligned}
& (u \oplus v)(t)=\sup _{s \in \mathbb{R}}\{u(s) \wedge v(t-s)\} t \in \mathbb{R} \\
& (u \odot v)(t)=\sup _{s \in \mathbb{R}}\{u(s) \wedge v(s-t)\} t \in \mathbb{R} \\
& (u \odot v)(t)=\sup _{\substack{s \in \mathbb{R} \\
s \neq 0}}\{u(s) \wedge v(t / s)\} t \in \mathbb{R} \\
& (u \oslash v)(t)=\sup _{s \in \mathbb{R}}\{u(s t) \wedge v(s)\} t \in \mathbb{R} .
\end{aligned}
$$

For $k \in \mathbb{R}^{+}, \quad k u$ is defined as $k u(t)=u(t / k)$ and $0 u(t)=\tilde{0}, \quad t \in \mathbb{R}$.

Some arithmetic operations for $\alpha$-level sets are defined as follows:

$u, v \in L(\mathbb{R})$ and $[u]_{\alpha}=\left[u_{\alpha}^{-}, u_{\alpha}^{+}\right]$and $[v]_{\alpha}=\left[v_{\alpha}^{-}, v_{\alpha}^{+}\right]$, $\alpha \in(0,1]$. Then

$$
\begin{gathered}
{[u \oplus v]_{\alpha}=\left[u_{\alpha}^{-}+v_{\alpha}^{-}, u_{\alpha}^{+}+v_{\alpha}^{+}\right]} \\
{[u \odot v]_{\alpha}=\left[u_{\alpha}^{-}-v_{\alpha}^{+}, u_{\alpha}^{+}-v_{\alpha}^{-}\right]} \\
{[u \odot v]_{\alpha}=\left[u_{\alpha}^{-} \cdot v_{\alpha}^{-}, u_{\alpha}^{+} \cdot v_{\alpha}^{+}\right]} \\
{[\tilde{1} \oslash u]_{\alpha}=\left[\frac{1}{u_{\alpha}^{+}}, \frac{1}{u_{\alpha}^{-}}\right] u_{\alpha}^{-}>0}
\end{gathered}
$$

For $u, v \in L(\mathbb{R})$, the supremum metric on $L(\mathbb{R})$ defined as

$$
D(u, v)=\sup _{0 \leq \alpha \leq 1} \max \left\{\left|u_{\alpha}^{-}-v_{\alpha}^{-}\right|,\left|u_{\alpha}^{+}-v_{\alpha}^{+}\right|\right\} .
$$

It is known that $D$ is a metric on $L(\mathbb{R})$, and $(L(\mathbb{R}), D)$ is a complete metric space.

A sequence $x=\left(x_{k}\right)$ of fuzzy numbers is said to be convergent to the fuzzy number $x_{0}$, if for every $\varepsilon>0$ there exists a positive integer $k_{0}$ such that $D\left(x_{k}, x_{0}\right)<\varepsilon$ for $k>k_{0}$ and a sequence $x=\left(x_{k}\right)$ of fuzzy numbers convergens to levelwise to $x_{0}$, iff $\lim _{k \rightarrow \infty}\left[x_{k}\right]_{\alpha}=\left[x_{0}\right]_{\alpha}^{-}$and $\lim _{k \rightarrow \infty}\left[x_{k}\right]_{\alpha}=\left[x_{0}\right]_{\alpha}^{+} \quad$ where $\quad\left[x_{k}\right]_{\alpha}=\left[\left(x_{k}\right)_{\alpha}^{-},\left(x_{k}\right)_{\alpha}^{+}\right] \quad$ and $\left[x_{0}\right]_{\alpha}=\left[\left(x_{0}\right)_{\alpha}^{-},\left(x_{0}\right)_{\alpha}^{+}\right]$for every $\alpha \in(0,1) .([2,6,11,12])$.

The statistical converge of fuzzy number was defined Savas [14] as follows;

A sequence $X=\left(X_{k}\right)$ of fuzzy numbers is said to be $\lambda$ statistically convergent to fuzzy numbers $X_{0}$ if every $\varepsilon>0$

$$
\lim _{n} \frac{1}{\lambda_{n}}\left|k \in I_{n}: d\left(X_{k}, X_{0}\right) \geq \varepsilon\right|=0 .
$$

Later many mathematicians such as Altınok, Altın and Et [1], J. A. Fridy and C. Orhan [10], F. Nuray [13] etc studied statistical convergence of fuzzy numbers.

Let $X$ be a vector space over $\mathbb{R}$, let $\|\|:. X \rightarrow L^{*}(\mathbb{R})$ and the mappings $L ; R$ (respectively, left norm and right norm) $:[0,1] \times[0,1] \rightarrow[0,1]$ be symetric, nondecreasing in both arguments and satisfy $L(0,0)=0$ and $R(1,1)=1$. ([8, 20])

The quadruple $(X,\|\|, L, R)$ is called fuzzy normed linear space (briefly $(X,\|\|) F N S$.$) and \|$.$\| a fuzzy norm if the$ following axioms are satisfied

1. $\|x\|=\tilde{0}$ iff $x=\theta$,

2. $\|r x\|=|r| \odot\|x\|$ for $x \in X, r \in \mathbb{R}$,

3. For all $x, y \in X$

a. $\|x+y\|(s+t) \geq L(\|x\|(s),\|y\|(t))$, whenever 
$s \leq\|x\|_{1}^{-}, t \leq\|y\|_{1}^{-}$and $s+t \leq\|x+y\|_{1}^{-}$,

b. $\|x+y\|(s+t) \leq R(\|x\|(s),\|y\|(t))$, whenever $s \geq\|x\|_{1}^{-}, t \geq\|y\|_{1}^{-}$and $s+t \geq\|x+y\|_{1}^{-}$.

Let $\left(X,\|\|_{C}\right)$ be an ordinary normed linear space. Then a fuzzy norm $\|$.$\| on X$ can be obtained

$$
\|x\|(t)=\left\{\begin{array}{cc}
0 & \text { if } 0 \leq t \leq a\|x\|_{C} \text { or } t \geq b\|x\|_{C} \\
\frac{t}{(1-a)\|x\|_{C}}-\frac{a}{1-a} & a\|x\|_{C} \leq t \leq\|x\|_{C} \\
\frac{-t}{(b-1)\|x\|_{C}}+\frac{b}{b-1} & \|x\|_{C} \leq t \leq b\|x\|_{C}
\end{array}\right.
$$

where $\|x\|_{C}$ is the ordinary norm of $x(\neq \theta), \quad 0<a<1$ and $1<b<\infty$. For $x=\theta$, define $\|x\|=\tilde{0}$. Hence $(X,\|\|$.$) is a$ fuzzy normed linear space.[8]

Sençimen [17] was defined convergence in fuzzy normed spaces by taking advantage of Kaleva [11, 8], as follows;

Let $(X,\|\|$.$) be an FNS. A sequence \left(x_{n}\right)_{n=1}^{\infty}$ in $X$ is convergent to $x \in X$ with respect to the fuzzy norm on $X$ and we denote by $x_{n} \stackrel{F N}{\rightarrow} x$ provided that $(D)-\lim _{n \rightarrow \infty}\left\|x_{n}-x\right\|=\tilde{0}$; i.e. for every $\varepsilon>0$ there is an $N(\varepsilon) \in \mathbb{N}$ such that $D\left(\left\|x_{n}-x\right\|, \tilde{0}\right)<\varepsilon$ for all $n \geq N(\varepsilon)$.

This means that for every $\varepsilon>0$ there is an $N(\varepsilon) \in \mathbb{N}$ such that

$$
\sup _{\alpha \in[0,1]}\left\|x_{n}-x\right\|_{\alpha}^{+}=\left\|x_{n}-x\right\|_{0}^{+}<\mathcal{E}
$$

for all $n \geq N(\varepsilon)$.

The purpose of this paper is to introduce and study a concept of lacunary convergence sequence with respect to fuzzy norm.

\section{Main Result}

In this section, we introduce notions of lacunary statistical convergence and lacunary summable in fuzzy normed linear spaces and we give some results.

\subsection{Definition}

Let $(X,\|\| \|)$ be an FNS and $\theta=\left\{k_{r}\right\}$ be a lacunary sequence. A sequence $x=\left(x_{k}\right)$ in $\mathrm{X}$ is said to be lacunary statistically convergent to $L \in X$ with respect to fuzzy norm on $X$ or $F S_{\theta}$ - convergent if for each $\varepsilon>0$

$$
\lim _{r \rightarrow \infty} \frac{1}{h_{r}}\left|\left\{k \in I_{r}: D\left(\left\|x_{k}-L\right\|, \tilde{0}\right) \geq \varepsilon\right\}\right|=0
$$

and we write $x_{k} \stackrel{F S_{\theta}}{\rightarrow}$ or $x_{k} \rightarrow L\left(F S_{\theta}\right)$ or $S_{\theta}{ }^{F N} \lim x_{k}=L$ where $I_{r}=\left(k_{r-1}, k_{r}\right]$.

This implies that for each $\varepsilon>0$, the set $K(\varepsilon)=\left\{k \in I_{r}:\left\|x_{k}-L\right\|_{0}^{+} \geq \varepsilon\right\}$ has natural density zero, namely, for each $\varepsilon>0,\left\|x_{k}-L\right\|_{0}^{+}<\varepsilon$ for a.a.k.

In this case we write $S_{\theta}{ }^{F N}-\lim x_{k}=L$. The set of all lacunary statistically convergent sequence w.r.t fuzzy norm on $\mathrm{X}$ will be denoted by $F S_{\theta}$.

$$
F S_{\theta}=\left\{x \text { : for some } \mathrm{L}, \mathrm{FS}_{\theta}-\lim x=L\right\} .
$$

\subsection{Definition}

Let $(X,\|\|$.$) be an FNS and \theta=\left\{k_{r}\right\}$ be a lacunary secuence. A sequence $x=\left(x_{k}\right)$ in $X$ is said to be lacunary summable with respect to fuzzy norm on $X$ if there is a $L \in X$ such that

$$
\lim _{r \rightarrow \infty} \frac{1}{h_{r}}\left(\sum_{k \in I_{r}} D\left(\left\|x_{k}-L\right\|, \tilde{0}\right)\right)=0
$$

where $I_{r}=\left(k_{r-1}, k_{r}\right]$ and $h_{r}=k_{r}-k_{r-1}$.

In this case we can write $x_{k} \rightarrow L\left(\left[N_{\theta}\right]_{F N}\right)$ and

$$
\left[N_{\theta}\right]_{F N}=\left\{x=\left(x_{k}\right): \lim _{r \rightarrow \infty} \frac{1}{h_{r}}\left(\sum_{k \in I_{r}} D\left(\left\|x_{k}-L\right\|, \tilde{0}\right)\right)=0, \text { for some } L\right\} .
$$

\subsection{Theorem}

Let $\theta=\left\{k_{r}\right\}$ be a lacunary sequence; then

1. a) $x_{k} \rightarrow L\left(\left[N_{\theta}\right]_{F N}\right) \Rightarrow x_{k} \rightarrow L\left(F S_{\theta}\right)$

b) $\left[N_{\theta}\right]_{F N}$ is a proper subset of $F S_{\theta}$

2. $x \in l_{\infty}$ and $x_{k} \rightarrow L\left(F S_{\theta}\right) \Rightarrow x_{k} \rightarrow L\left(\left[N_{\theta}\right]_{F N}\right)$

3. $F S_{\theta} \cap l_{\infty}=\left[N_{\theta}\right]_{F N} \cap l_{\infty}$, where $l_{\infty}$ is the set of bounded sequences.

Proof.

(1) a) If $\varepsilon>0$ and $x_{k} \rightarrow L\left(\left[N_{\theta}\right]_{F N}\right)$ then

$$
\begin{aligned}
\sum_{k \in I_{r}} D\left(\left\|x_{k}-L\right\|, \tilde{0}\right) & \geq \sum_{\substack{k \in I_{r} \\
D\left(\left\|x_{k}-L\right\|, \tilde{)}>\varepsilon\right.}} D\left(\left\|x_{k}-L\right\|, \tilde{0}\right) \\
& \geq \varepsilon \cdot\left\{\left\{k \in I_{r}: D\left(\left\|x_{k}-L\right\|, \tilde{0}\right) \geq \varepsilon\right\} .\right.
\end{aligned}
$$

So we can write

$$
\frac{1}{h_{r}}\left|\left\{k \in I_{r}: D\left(\left\|x_{k}-L\right\|, \tilde{0}\right) \geq \varepsilon\right\}\right| \leq \frac{1}{h_{r}} \sum_{k \in I_{r}} D\left(\left\|x_{k}-L\right\|, \tilde{0}\right)
$$




$$
\Rightarrow \lim _{r} \frac{1}{h_{r}}\left|\left\{k \in I_{r}: D\left(\left\|x_{k}-L\right\|, \tilde{0}\right) \geq \varepsilon\right\}\right|=0 .
$$

This imply $x_{k} \rightarrow L\left(F S_{\theta}\right)$.

b) In order to indicate that the inclusion $\left[N_{\theta}\right]_{F N} \subseteq F S_{\theta}$ in (1) is proper, let $\theta$ be given and define as

$$
x_{k}= \begin{cases}1,2, \ldots,\left[\sqrt{h_{r}}\right] & ; \text { at the first }\left[\sqrt{h_{r}}\right] \text { integers in } \mathrm{I}_{r} \\ 0 & ; \text { otherwise. }\end{cases}
$$

Note that $x$ is not bounded.

We have, for every $\varepsilon>0$,

$$
\frac{1}{h_{r}}\left\{k \in I_{r}: D\left(\left\|x_{k}-0\right\|, \tilde{0}\right) \geq \varepsilon\right\} \mid=\frac{\sqrt{h_{r}}}{h_{r}} \rightarrow 0
$$

as $r \rightarrow \infty$.

i.e., $x_{k} \rightarrow 0\left(F S_{\theta}\right)$. On the other hand

$$
\begin{aligned}
\frac{1}{h_{r}} \sum_{k \in I_{r}} D\left(\left\|x_{k}-0\right\|, \tilde{0}\right) & =\frac{1}{h_{r}} \sum_{k \in I_{r}}\left\|x_{k}\right\|_{0}^{+} \\
& =\frac{1}{h_{r}} \cdot \frac{\left[\sqrt{h_{r}}\right] \cdot\left(\left[\sqrt{h_{r}}\right]+1\right)}{2} \rightarrow \frac{1}{2} \neq 0
\end{aligned}
$$

hence $x_{k} \nrightarrow 0\left(\left[N_{\theta}\right]_{F N}\right)$.

(2) Suppose that $x_{k} \rightarrow L\left(F S_{\theta}\right)$ and $x \in l_{\infty}$. Then we say $D\left(\left\|x_{k}-L\right\|, \tilde{0}\right)<M$ for all k. Given $\varepsilon>0$, we get

$$
\frac{1}{h_{r}} \sum_{k \in I_{r}} D\left(\left\|x_{k}-L\right\|, \tilde{0}\right)
$$$$
=\frac{1}{h_{r}} \sum_{\substack{k \in I_{r} \\ D\left(\left\|x_{k}-L\right\|, \tilde{0}\right) \geq \varepsilon}} D\left(\left\|x_{k}-L\right\|, \tilde{0}\right)+\frac{1}{h_{r}} \sum_{\substack{k \in I_{r} \\ D\left(\|\|_{k}-L, \tilde{0}\right)<\varepsilon}} D\left(\left\|x_{k}-L\right\|, \tilde{0}\right)
$$$$
\leq \frac{M}{h_{r}} \cdot\left|\left\{k \in I_{r}: D\left(\left\|x_{k}-L\right\|, \tilde{0}\right) \geq \varepsilon\right\}\right|+\varepsilon \text {. }
$$

From hence $x_{k} \rightarrow L\left(\left[N_{\theta}\right]_{F N}\right)$.

If we pay attention here, the example given in (1) we have got an unbounded sequence. Afterwards we added boundedness in (2). In this case the hypothesis given (1) for the bounded sequence already provide.

(3) When the boundedness condition is added as a result of (1) and (2), any $\left[N_{\theta}\right]_{F N}$ - summable sequence is $F S_{\theta}$ - convergence. At the same time any $F S_{\theta}-$ convergent sequence is $\left[N_{\theta}\right]_{F N}-$ summable sequence.

\subsection{Lemma}

For any lacunary sequence $\theta, F S-\lim x=L$ implies $F S_{\theta}-\lim x=L$ if and only if $\liminf _{r} q_{r}>1$.

Proof.
Suppose that $\liminf _{r} q_{r}>1$. Then there exists a $\delta>0$ such that $q_{r}>1+\delta$ for sufficiently large $r$, which implies that $\frac{h_{r}}{k_{r}} \geq \frac{\delta}{1+\delta}$.

If $x_{k} \rightarrow L(F S)$, then for every $\varepsilon>0$ and sufficiently large $\mathrm{r}$, we have

$$
\begin{aligned}
& \frac{1}{h_{r}}\left|\left\{k \leq k_{r}: D\left(\left\|x_{k}-L\right\|, \tilde{0}\right) \geq \varepsilon\right\}\right| \\
& \geq \frac{1}{k_{r}}\left\{\left\{k \in I_{r}: D\left(\left\|x_{k}-L\right\|, \tilde{0}\right) \geq \varepsilon\right\} \mid\right. \\
& \geq \frac{\delta}{1+\delta} \cdot \frac{1}{h_{r}}\left|\left\{k \in I_{r}: D\left(\left\|x_{k}-L\right\|, \tilde{0}\right) \geq \varepsilon\right\}\right| .
\end{aligned}
$$

So this result indicates that $x$ is lacunary statistical convergence with respect to fuzzy norm.

\subsection{Lemma}

For any lacunary sequence $\theta, F S-\lim x=L$ implies $F S_{\theta}-\lim x=L$ if and only if $\limsup _{r} q_{r}<\infty$.

\section{Proof.}

If $\limsup _{r} q_{r}<\infty$, then there is a $H>0$ such that $q_{r}<H$ for all $r$. Suppose that $x_{k} \rightarrow L\left(F S_{\theta}\right)$ and let $F N_{r}=\left|\left\{k \in I_{r}: D\left(\left\|x_{k}-L\right\|, \tilde{0}\right) \geq \varepsilon\right\}\right|$. By (8), given $\varepsilon>0$, there is an $r_{0} \in \mathbb{N}$ such that

$$
\frac{F N_{r}}{h_{r}}<\mathcal{E} \text { for all } r>r_{0}
$$

Now let $M=\max \left\{F N_{r}: 1 \leq r \leq r_{0}\right\}$ and let $n$ be any integer satisfying $k_{r-1}<n \leq k_{r}$; then we can write

$$
\begin{aligned}
\frac{1}{n} \mid\{k \leq n & \left.: D\left(\left\|x_{k}-L\right\|, \tilde{0}\right) \geq \varepsilon\right\}\left|\leq \frac{1}{k_{r-1}}\right|\left\{k \leq k_{r}: D\left(\left\|x_{k}-L\right\|, \tilde{0}\right) \geq \varepsilon\right\} \mid \\
& =\frac{1}{k_{r-1}}\left\{F N_{1}+F N_{2}+\ldots+F N_{r_{0}}+F N_{r_{0}+1}+\ldots+F N_{r}\right\} \\
& \leq \frac{M}{k_{r-1}} \cdot r_{0}+\frac{1}{k_{r-1}} \cdot\left\{h_{r_{0}+1} \cdot \frac{F N_{r_{0}+1}}{h_{r_{0}+1}}+\ldots+h_{r} \cdot \frac{F N_{r}}{h_{r}}\right\} \\
& \leq \frac{r_{0} \cdot M}{k_{r-1}}+\frac{1}{k_{r-1}} \cdot \sup _{r>r_{0}} \frac{F N_{r}}{h_{r}}\left\{h_{r_{0}+1}+\ldots+h_{r}\right\} \\
& \leq \frac{r_{0} \cdot M}{k_{r-1}}+\varepsilon \cdot \frac{k_{r}-k_{r_{0}}}{k_{r-1}} \text { by } \\
& \leq \frac{r_{0} \cdot M}{k_{r-1}}+\varepsilon \cdot q_{r} \\
& \leq \frac{r_{0} \cdot M}{k_{r-1}}+e \cdot H .
\end{aligned}
$$

Hence we can say that $x$ is lacunary statistical 
convergence with respect to fuzzy norm.

\subsection{Theorem}

Let $\theta$ be a lacunary secuence; then $F S=F S_{\theta}$ if and only if

$$
1<\liminf _{r} q_{r} \leq \lim _{r} \sup q_{r}<\infty
$$

then $F S-\lim x=L$ implies $F S_{\theta}-\lim x=L$.

\section{Conclusion}

In this study, we introduced the concept of lacunary statistical convergence with respect to a fuzzy norm. We also studied the relation between lacunary summabilty and lacunary convergence in fuzzy normed space. As a result, we have seen that results similar to the results in classical normed spaces are also obtained in fuzzy normed spaces.

\section{References}

[1] H. Altınok, Y. Altın, and M. Et, Lacunary almost statistical convergence of fuzzy numbers, Thai Journal of Mathematics, vol. 2, no. 2, pp. 265-274, 2004.

[2] T. Bag and SK. Samanta, Fixed point theorems in Felbin's type fuzzy normed linear spaces, J. Fuzzy Math., vol. 16, no. 1, (2008), pp. 243-260.

[3] M. Et; M. Çınar and M. Karakaş, On $\lambda$ - statistical convergence of order $\alpha$ of sequences of function, J. Inequal. Appl. 2013, 2013:204, pp. 1-8.

[4] R. Çolak, On $\lambda$ - statistical convergence, Conference on Summability and Applications, May 12-13, 2011, Istanbul Turkey.

[5] J. S. Connor, The statistical and strong $p$-Cesaro convergence of sequences, Analysis 8 (1988), pp. 47-63.

[6] P. Diamond and P. Kloeden, Metric Spaces of Fuzzy Sets-Theory and Applications. World Scientific Publishing, Singapore, 1994.
[7] H. Fast, Sur la convergence statistique, Colloq. Math. 2 (1951), 241-244.

[8] C. Felbin, Finite-dimensional fuzzy normed linear space, Fuzzy Sets and Systems 48(2) (1992), 239-248.

[9] J. Fridy, On statistical convergence, Analysis 5 (1985), 301-313.

[10] J. A. Fridy and C. Orhan, Lacunary Statistical Convergence, Pasific Journal of Mathematics, vol. 160, no. 1, 43-51, 1993.

[11] O. Kaleva and S. Seikkala, On fuzzy metric spaces, Fuzzy Sets and Systems 12(3) (1984), 215-229.

[12] M. Mizumoto and K. Tanaka, Some properties of fuzzy numbers, Advances in Fuzzy Set Theory and Applications, pp. 153-164, North-Holland, Amsterdam, 1979.

[13] F. Nuray, Lacunary Statistical Convergence of Sequence of Fuzzy Numbers, Fuzzy Sets and Systems, 99 (1998) 353,355.

[14] E. Savas, On Strongly $\lambda$ - summable Sequences of Fuzzy Numbers, Information Science, (125) (1-2), (2000).

[15] I. J. Schoenberg, The integrability of certain functions and related summability methods, Amer. Math. Monthly 66 (1959), 361-375.

[16] A. R. Freedman, J. J. Sember and M. Raphael, Some Cesaro type summability spaces, Proc. London Math. Soc., 37 (1978), 508-520.

[17] C. Sencimen and S. Pehlivan, Statistical convergence in fuzzy normed linear spaces, Fuzzy Sets and Systems 159(3) (2008), 361-370.

[18] H. Steinhaus, Sur la convergence ordinaire et la convergence asymptotique, Colloq. Math. 2 (1951), 73-74.

[19] T. Šalát (1980) On statistically convergent sequences of real numbers, Math. Slovaca 30, 139-150.

[20] J. Xiao and X. Zhu, On linearly topological structure and property of fuzzy normed linear space. Fuzzy Sets and Systems 125(2) (2002), 153-161.

[21] L. A. Zadeh, Fuzzy sets, Information and Control 8 (1965), 338-353. 